\title{
Targeting the A2A adenosine receptor counteracts immunosuppression in vivo in a mouse model of chronic lymphocytic leukemia
}

\section{Francesca Arruga, ${ }^{1}$ Sara Serra, ${ }^{1}$ Nicoletta Vitale,${ }^{1}$ Giulia Guerra, ${ }^{1}$ Andrea Papait, ${ }^{1}$ Benjamin Baffour Gyau, ${ }^{1}$ Francesco Tito, ${ }^{1}$ Dimitar Efremov, ${ }^{2}$ Tiziana Vaisitti ${ }^{1 *}$ and Silvia Deaglio ${ }^{1 *}$}

${ }^{1}$ Laboratory of Cancer Immunogenetics, Department of Medical Sciences, University of Turin, Turin and ${ }^{2}$ International Center for Genetic Engineering and Biotechnology, Trieste, Italy

${ }^{\#} T V$ and SD contributed equally as co-senior authors.

\section{ABSTRACT}

T umor immunosuppression is a major cause of treatment failure and disease relapse, both in solid tumors and leukemia. Local hypoxia is among the conditions that cause immunosuppression, acting at least in part through the upregulation of extracellular adenosine levels, which potently suppress T-cell responses and skew macrophages towards an M2 phenotype. Hence, there is intense investigation to identify drugs that target this axis. By using the TCL1 adoptive transfer chronic lymphocytic leukemia mouse model, we show that adenosine production and signaling are upregulated in the hypoxic lymphoid niches, where intense colonization of leukemic cells occurs. This leads to a progressive remodeling of the immune system towards tolerance, with expansion of $\mathrm{T}$ regulatory cells (Treg), loss of $\mathrm{CD}^{+} \mathrm{T}$-cell cytotoxicity and differentiation of murine macrophages towards the patrolling (M2-like) subset. In vivo administration of SCH58261, an inhibitor of the A2A adenosine receptor, re-awakens T-cell responses, while limiting Treg expansion, and re-polarizes monocytes towards the inflammatory (M1-like) phenotype. These results show for the first time the in vivo contribution of adenosine signaling to immune tolerance in chronic lymphocytic leukemia, and the translational implication of drugs interrupting this pathway. Although the effects of SCH58261 on leukemic cells are limited, interfering with adenosine signaling may represent an appealing strategy for combination-based therapeutic approaches.

\section{Introduction}

Chronic lymphocytic leukemia (CLL) is a slowly progressive disease characterized by complex interactions between leukemic cells and surrounding elements occurring mainly in privileged niches, such as lymphoid organs. ${ }^{1-3}$ Over the years, microenvironment-activated pathways, essential to sustain and protect leukemic cells, became a target for novel therapeutic approaches aimed at interrupting these interactions. ${ }^{4,5}$ However, despite their impressive clinical benefits, it is becoming clear that these drugs fail to produce a cure, underlining the need for combination strategies. ${ }^{67}$ We now know that the cross-talk between CLL cells and the microenvironment is not a one-way relationship, in which leukemic cells "passively" receive positive stimuli. Several pieces of evidence showed that CLL cells play an active role in remodeling the phenotype and functions of cellular elements that inhabit the lymphoid niche. ${ }^{8}$ For example, nurse-like cells, an M2-like macrophage subset known to be a CLL-supporting population, can be differentiated only in the presence of leukemic B cells, suggesting that CLL cells directly shape the microenvironment towards tumor-friendly immuno-tolerant conditions. ${ }^{9}$ Over the last two decades, many co-stimulatory signals and circuits have been identified, building the picture of a highly articulated space in which it may be difficult to understand hierarchical organization. It is therefore important to unravel unifying structural conditions of the microenvironment that may be more amenable to therapeutic targeting than single molecular circuits.
Haematologica 2021

Volume 106(5):1343-1353

\section{Correspondence:}

SILVIA DEAGLIO

silvia.deagli@@unito.it

Received: November 18, 2019.

Accepted: April 9, 2020.

Pre-published: April 16, 2020.

https://doi.org/10.3324/haematol.2019.242016

(C)2021 Ferrata Storti Foundation

Material published in Haematologica is covered by copyright. All rights are reserved to the Ferrata Storti Foundation. Use of published material is allowed under the following terms and conditions:

https://creativecommons.org/licenses/by-nc/4.0/legalcode. Copies of published material are allowed for personal or internal use. Sharing published material for non-commercial purposes is subject to the following conditions: https://creativecommons.org/licenses/by-nc/4.0/legalcode, sect. 3. Reproducing and sharing published material for commercial purposes is not allowed without permission in writing from the publisher. 
Hypoxia, defined as a condition of low oxygen tension, is a feature of the tumor microenvironment, particularly, but not exclusively, of solid tumors. ${ }^{10,11}$ Hypoxia activates a transcriptional program ruled by hypoxia-inducible factor (HIF)-1 $\alpha$, which contributes to building local conditions that shield the tumor from eventual therapies and that blunt immune responses. ${ }^{12,13}$ Our previous data indicate that the CLL lymph node is a hypoxic environment and that hypoxia acts, at least in part, through the activation of extracellular adenosine production and signaling. ${ }^{14}$

This work shows the modulation of adenosine signaling and the effects of its blockade in the CLL microenvironment using the TCL1 adoptive transfer model, making it a suitable option for combination strategies.

\section{Methods}

\section{Adoptive transfer TCL1 mouse model}

Ten million cells from different TCL1 leukemic clones were intraperitoneally injected (in $200 \mu \mathrm{L}$ of phosphate-buffered saline) in 8-week-old C57BL/6 female mice and left to engraft. Where indicated, mice were treated with SCH58261 (Selleckchem) administered intraperitoneally at a dose of $1 \mathrm{mg} / \mathrm{kg}$ every other day. Treatment was started blindly 4 days after injection of leukemic cells, following published protocols. ${ }^{15}$ When showing clear signs of disease or at the end of the treatment schedule, mice were euthanized and organs (peritoneal cavity, spleen, peripheral blood and bone marrow) collected. In experiments aimed at evaluating progressive hypoxia, mice were intraperitoneally injected with $100 \mathrm{mg} / \mathrm{kg}$ pimonidazole hydrochloride (PIM; Hypoxyprobe, Inc.) $2 \mathrm{~h}$ before euthanasia. All the in vivo experiments were performed following the institutional and national guidelines and approved by the Italian Ministry of Health (authorization $n$. 118/2018-PR).

\section{Antibodies and reagents}

A complete list of antibodies used in flow cytometry is reported in Online Supplementary Table S1.

\section{Immunohistochemistry and confocal microscopy}

Formalin-fixed paraffin-embedded spleen sections were stained and analyzed as previously reported.$^{14}$ Full details are provided in the Online Supplementary Information.

\section{Detection of hypoxia using pimonidazole hydrochloride}

Cells purified from mice injected with PIM prior to euthanasia, were fixed, permeabilized and stained with a FITC-conjugated mouse anti-PIM antibody (Hypoxyprobe, Inc). Fluorescence was analyzed by flow cytometry. Confocal analyses of PIM staining were performed as described in the Online Supplementary Information.

\section{Real-time polymerase chain reaction}

Quantitative real-time polymerase chain reaciont (qRT-PCR) was performed using the 7900 HT Fast Real Time PCR System (SDS 2.3 software). Primers for Entpd1 (Mm00515447_m1), Nt5e (Mm00501920_m1), Adora2a (Mm00802075_m1), Adora2b (Mm00839292_m1), Adora3 (Mm00802076_m1), Ada (Mm00545720_m1), and Actb (Mm02619580_g1) were from Life Technologies.

\section{High performance liquid chromatography measurement of adenosine production}

Nucleotide metabolism was analyzed by high-performance liq- uid chromatography. ${ }^{16}$ A full description of the methods can be found in the Online Supplementary Information.

\section{Statistical analyses}

Data are reported as box plots, with the top and bottom of the rectangle defining the third and first quartile, respectively, and the whiskers defining the maximum and minimum values. The line inside the rectangle represents the median value. Data were analyzed with GraphPad Prism v6 software (GraphPad Software). Differences were considered statistically significant when $P$-values were $\leq 0.05$. Results are shown as means \pm standard deviation. Statistics were calculated using either the Mann-Whitney test for unpaired data or the Wilcoxon test for paired data, as specified for each graph in the figure legends.

Methods described fully in the Online Supplementary Information include the enzyme-linked immunosorbent assay for protein kinase A (PKA) activity, assays of cell degranulation and cytokines release, as wells as the Phosflow and apoptosis assays.

\section{Results}

\section{Leukemia engraftment is accompanied by progressive hypoxia}

The TCL1 adoptive transfer model is based on the intraperitoneal injection of leukemic cells derived from TCL1 transgenic mice into normal immunocompetent C57BL/6 recipients. Rapid disease engraftment occurs in $100 \%$ of cases: after a period ranging from 2 to 5 weeks, depending on the number of cells transferred and on the leukemic clone, B220+/CD5 $5^{+}$leukemic cells appear in the peripheral blood (Figure 1A). At this moment, the spleen already shows signs of leukemic colonization, with large nodules progressively effacing the normal tissue architecture (Figure 1B). Terminally ill animals show signs of widespread disease, with massive colonization of the spleen, bone marrow, peritoneal cavity and peripheral blood. At this stage, no architecture is recognizable in the spleen, which is composed of a sheet of cells, with significant necrosis (Figure 1B). Following the hypothesis that leukemic colonization is accompanied by the progressive onset of hypoxic conditions, we stained for carbonic anhydrase IX (CAIX), an enzyme that is under the direct transcriptional control of HIF-1 $\alpha$, the master regulator of hypoxia. Images show a gradient of staining intensity from wild-type (WT) to terminally ill mice (Figure 1B). To confirm this observation, we injected mice with PIM, a compound that forms stable adducts with cellular macromolecules under low oxygen tension. Triple staining with anti-B220, anti-CD3 and anti-PIM antibodies confirmed that the spleen of terminally ill mice was intermixed with $\mathrm{B}$ and $\mathrm{T}$ cells that were positive for PIM (Figure 1C). Consistently, staining purified B cells from the spleen, peripheral blood and bone marrow with an anti-PIM antibody demonstrated a marked increase of $\mathrm{PIM}^{+}$cells in leukemic compared to normal B lymphocytes (Figure 1D).

\section{Upregulation of the adenosine-producing capacity of leukemic cells during leukemia progression}

Our previous data indicate that hypoxia acts, at least using in vitro culture systems, through the upregulation of an extracellular enzymatic system that dismantles ATP and ADP, generating adenosine. ${ }^{14}$ We then sought to determine expression levels and activity of the adenosinergic axis during disease progression. 
A

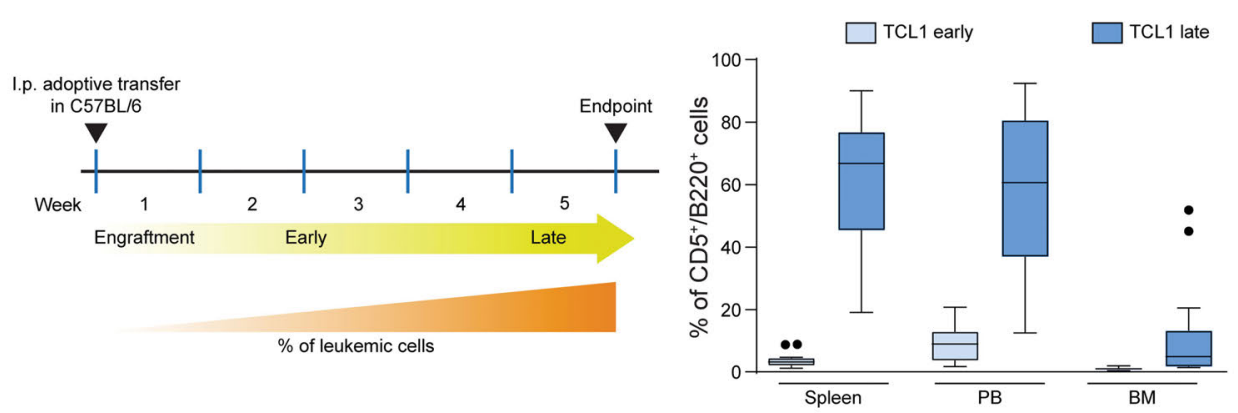

B

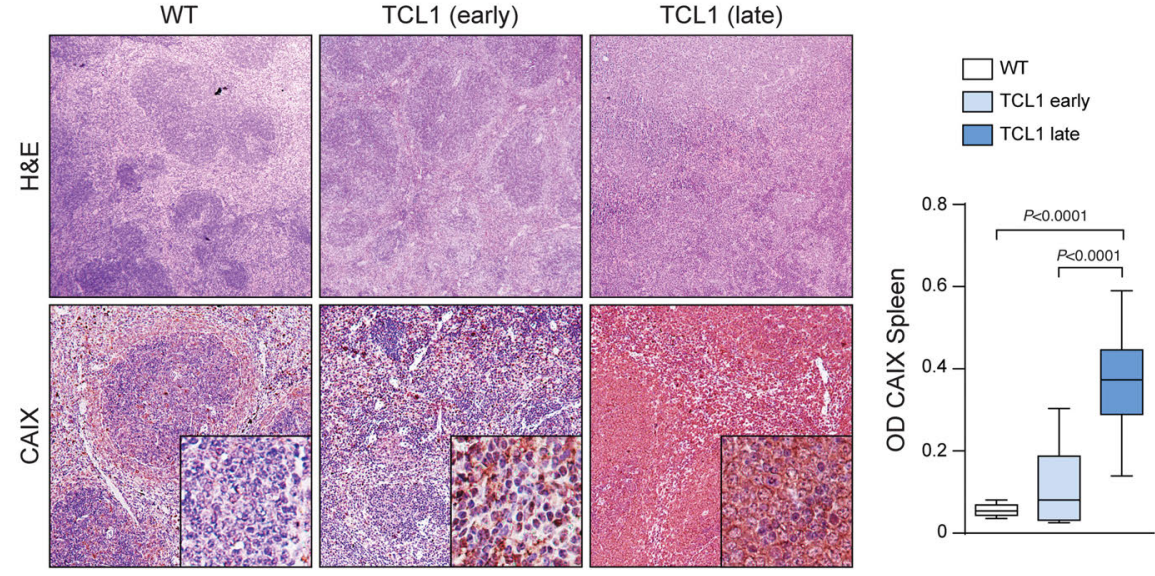

C
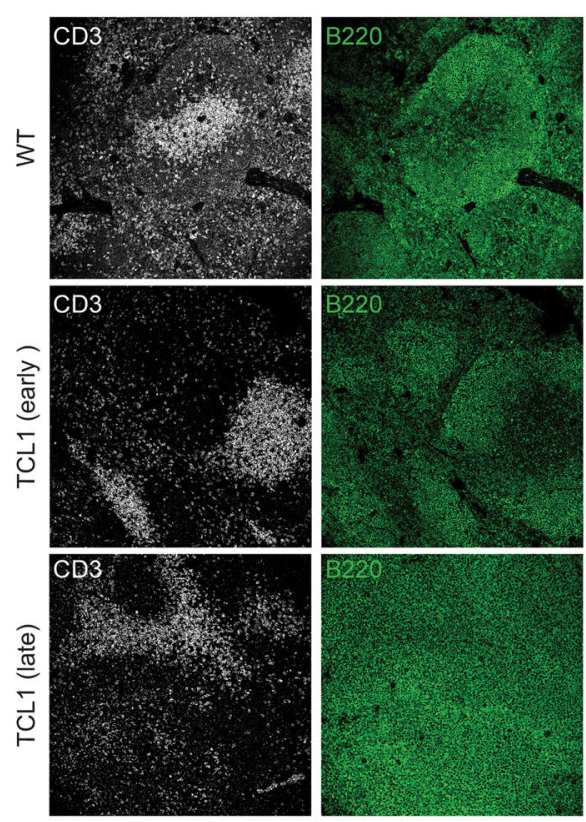
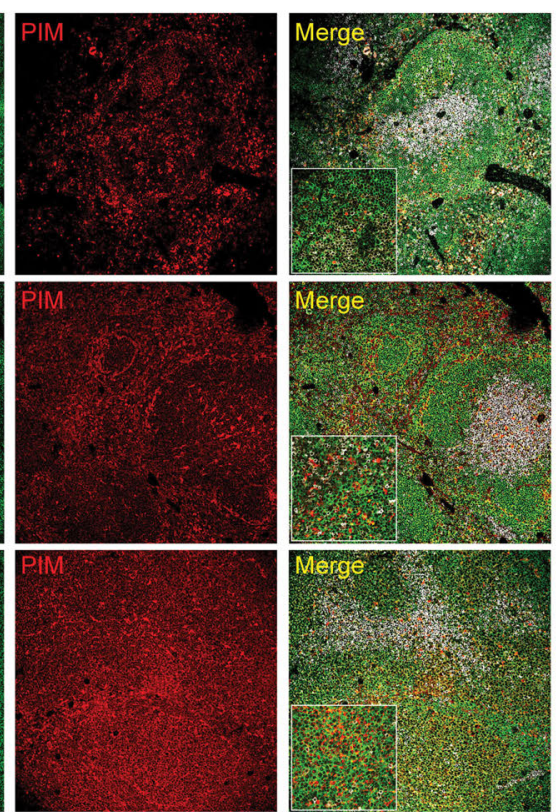

D
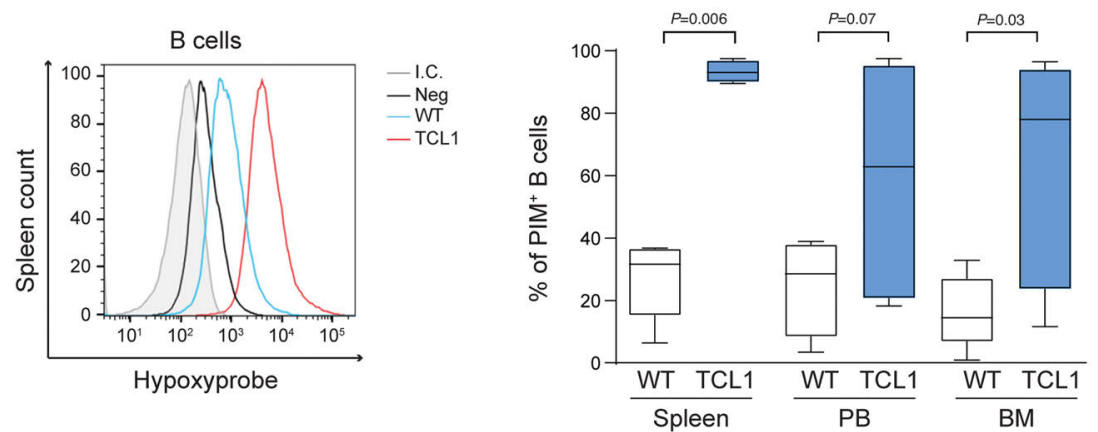

Figure 1. Leukemia engraftment is accompanied by progressive hypoxia. (A) Left panel: schematic representation of the experimental setting and schedule; right panel: box plot of the percentages of leukemic cells traced in the different engrafted districts early and late after intraperitoneal (i.p.) injection of leukemic cells in C57BL/ 6 mice $(n=20$ mice each time point). PB: peripheral blood; BM: bone marrow. (B) Representative immunohistochemical analysis of tissue architecture (top; hematoxylin and eosin, $\mathrm{H \& E}$ ) and of a progressively hypoxic environment (bottom; carbonic anhydrase IX, CAIX) in spleen sections at different stages of disease progression compared to wild-type (WT) spleen. Magnification 20X, magnification of inset 40X. The graph on the right summarizes quantification of CAIX intensity (WT mice $n=7$; TCL1 early and late timepoints $n=13$ ). Statistics were calculated with the Mann-Whitney test for unpaired data. (C) Confocal microscopy analysis of pimonidazole (PIM; red) accumulation in spleen sections at different stages of leukemia engraftment compared to WT spleen. B220 (green) and CD3 (white) mark $B$ and $T$ cells, respectively. Magnification 20X, insert 63X. (D) Left panel: representative plot of flow cytometry analysis of PIM accumulation in B cells collected from spleen. Right panel: cumulative graph of PIM staining on B cells from spleen, PB and BM samples of TCL1-injected mice $(n=7)$ compared to not injected WT mice $(n=7)$. Statistics were calculated with the MannWhitney test for unpaired data. 
A

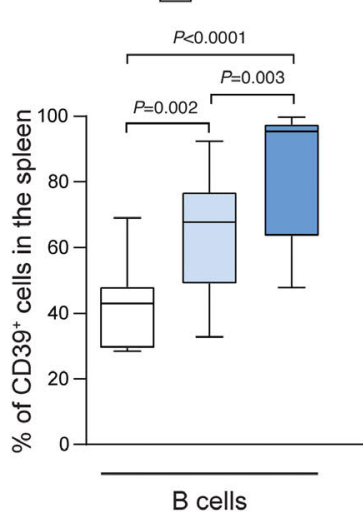

B

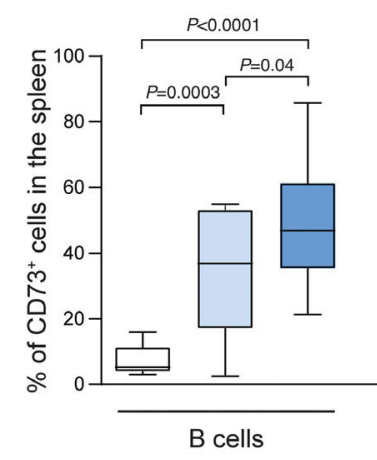

$\square$ TCL1 early

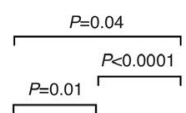


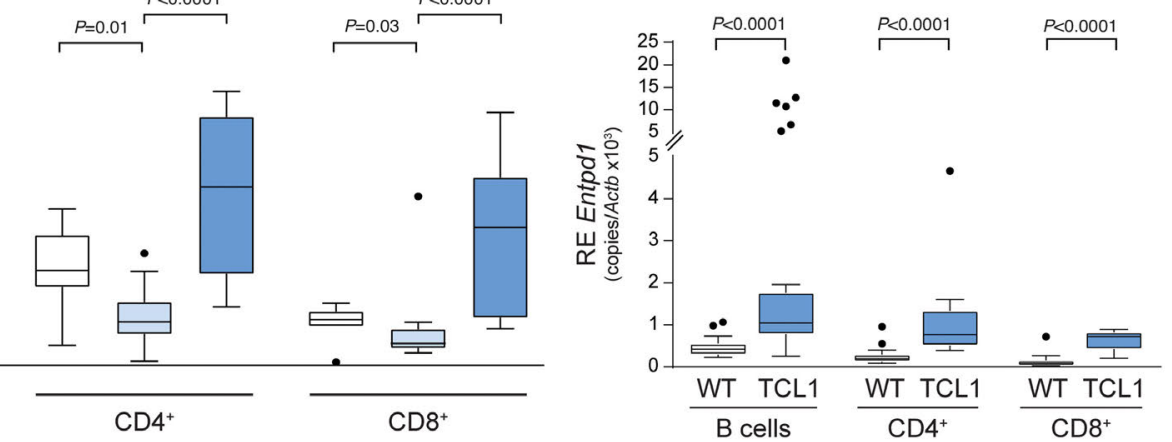

C
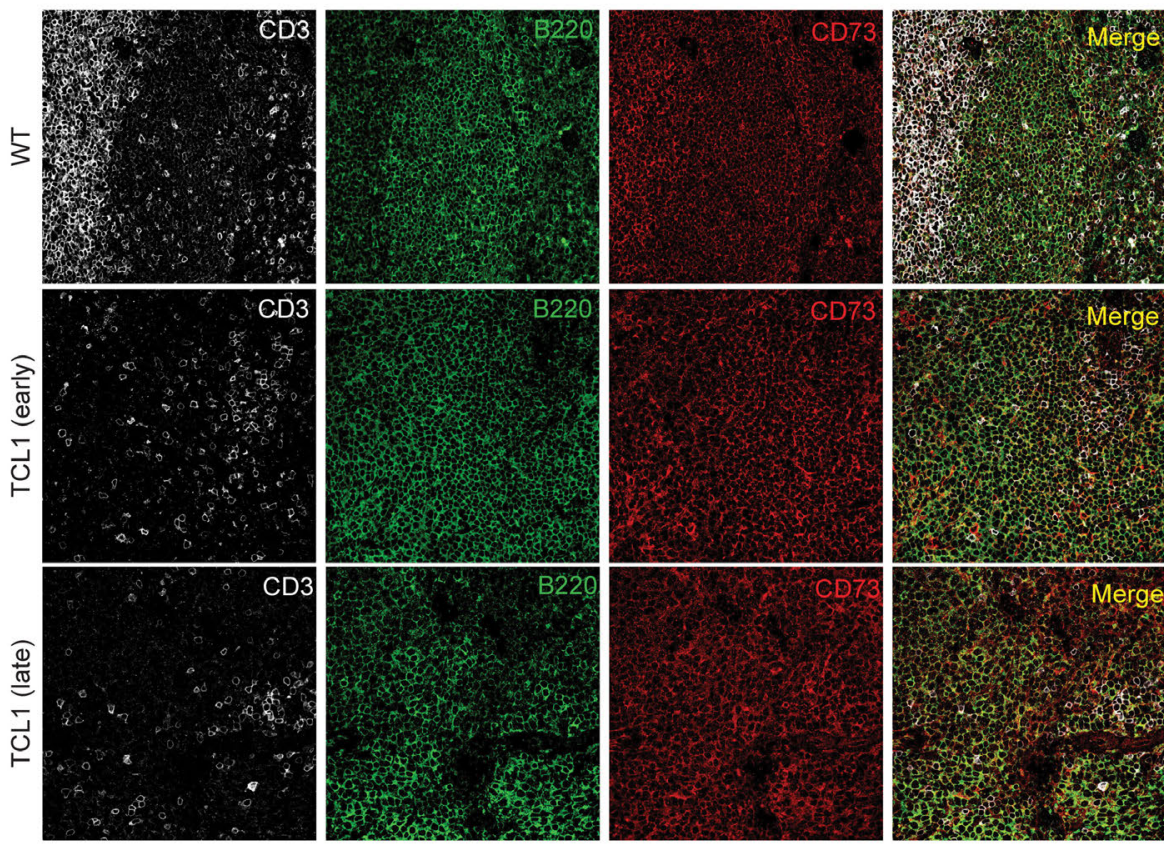

Figure 2. Upregulation of adenosine-generating enzymes during leukemia progression. (A) Left panel. Flow cytometry analysis of surface CD39 expression on B cells, and $\mathrm{CD}^{+}$and $\mathrm{CD} 8^{+} \mathrm{T}$ cells collected from spleens of TCL1 mice at early and late time-points ( $n=20$, each time-point) after leukemia injection and compared to expression in wild-type (WT) mice $(n=7)$. Right panel. Quantitative real-time polymerase chain reaction (qRT-PCR) analysis of Entpd1 expression in terminally ill TCL1 mice $(n=20)$ compared to WT animals $(n=20)$. Statistics were calculated with the Mann-Whitney test for unpaired data. RE: relative expression. (B) Left panel. Flow cytometry analysis of surface CD73 expression on B cells, and CD4 ${ }^{+}$and $C D 8^{+}$T cells collected from spleens of TCL1 mice at early and late time points ( $n=20$, each time point) after leukemia injection and compared to WT mice $(n=7)$. Right panel. qRT-PCR analysis of Nt5e expression in terminally ill TCL1 mice ( $n=20)$ compared to WT animals $(n=20)$. Statistics were calculated with the Mann-Whitney test for unpaired data. RE: relative expression. (C) Confocal microscopy analysis of CD73 (red) staining in spleen sections from TCL1 and WT mice. B220 (green) and CD3 (white) mark B and T cells, respectively. Magnification 63X. 
A


B

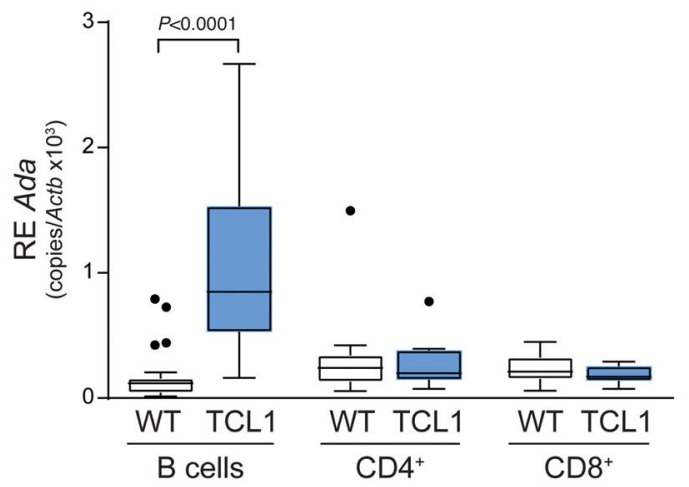

Figure 3. Upregulation of adenosine-producing capacity during leukemia progression. (A) Top panels. A high-performance liquid chromatography profile of inosine (INO) and adenosine (ADO) peaks at baseline or upon AMP supply in the presence or absence of the indicated doses of the adenosine deaminase inhibitor EHNA. Bottom panels. Cumulative graphs of INO and ADO concentrations in the indicated experimental conditions in five independent experiments. Statistics were calculated using the Wilcoxon test for paired data. (B) Quantitative real-time polymerase chain reaction analysis of $A d a$ expression in $\mathrm{B}$ cells and $\mathrm{CD}^{+}$or $\mathrm{CD}^{+} \mathrm{T}$ lymphocytes of TCL1 mice $(n=20)$ compared to the expression of wild-type (WT) animals $(n=20)$. Statistics were calculated with the Mann-Whitney test for unpaired data. RE: relative expression.

The first observation was that CD39, the enzyme that converts ATP or ADP to AMP, and CD73, the enzyme that removes the last phosphate generating adenosine, were significantly upregulated in leukemic B cells from eight different TCL1 leukemias. This was evident by flow cytometry (Figure 2A and B) and confocal microscopy (for CD73 only) (Figure 2C) performed at early and late time points, and confirmed by qRT-PCR performed on cells collected from spleens of terminally ill animals (Figure 2A and B). Cells from peripheral blood and bone marrow showed the same behavior (Online Supplementary Figure S1A and $B$ ). Conversely, only CD39 was increased both at the mRNA and protein levels on $\mathrm{CD}^{+}$and $\mathrm{CD} 8^{+} \mathrm{T}$ cells collected from spleen, peripheral blood and bone marrow of leukemic mice, compared to WT mice. In these cells, CD73 was transcriptionally stable although we observed slight but significant downregulation of surface protein expression (Figure 2A and B, Online Supplementary Figure $S 1 A$ and $B)$.

Upregulation of ATP-dismantling enzymes is consistent with an enhanced adenosine-producing capability of the leukemic microenvironment, which was in fact documented using high-performance liquid chromatography to determine adenosine production by leukemic B cells. For these studies, we used purified leukemic cells from terminally ill mice and incubated them with AMP, the substrate of CD73, which controls the last step in the reaction and measured adenosine and inosine accumulation. Results showed rapid and efficient consumption of AMP, with conversion to inosine (Figure 3A), the final degradation product, indicating that adenosine is metabolized by adenosine deaminase into inosine. In agreement, (i) the use of inhibitors of adenosine deaminase - erythro-9-(2hydroxy-3-nonyl)adenine (EHNA) - prevented inosine generation, leading to the appearance of an adenosine peak following incubation with AMP (Figure 3A) and (ii) qRT-PCR data indicated increased adenosine deaminase expression by the leukemic subset (Figure 3B). These results are in line with what was observed for human CLL in which hypoxia induced accumulation of inosine by leukemic cells, suggesting that under hypoxia this cell population actively scavenges extracellular nucleotides.

\section{Upregulation of the adenosine-sensing ability during leukemia progression}

According to our working hypothesis, hypoxia would boost not only adenosine production, but also adenosine sensing in the leukemic microenvironment. To investigate 
this, we checked the expression of adenosine receptors in cells residing in the leukemic microenvironment. The qRT-PCR data from cell subpopulations showed marked upregulation of the $\mathrm{A} 2 \mathrm{~A}$ adenosine receptor in leukemic $\mathrm{B}$-cell and $\mathrm{CD}^{+}$and $\mathrm{CD}^{+} \mathrm{T}$-cell subsets (Figure 4A). The other two adenosine receptors, i.e., A2B and A3, followed a similar trend, even though expression levels remained extremely low (Online Supplementary Figure S2A and B). Macrophages could not be examined by qRT-PCR because of their extremely low numbers that prevented purification. However, immunohistochemistry performed on sections of spleens from terminally ill animals confirmed a general increase in the expression of $\mathrm{A} 2 \mathrm{~A}$ in this tissue (Figure 4B).

A2A are G-protein-coupled receptors that lead to accumulation of cAMP, in turn activating PKA and ultimately inhibiting the pro-inflammatory action of the nuclear factor- $\mathrm{\kappa B}$ pathway through phosphorylation of cAMP
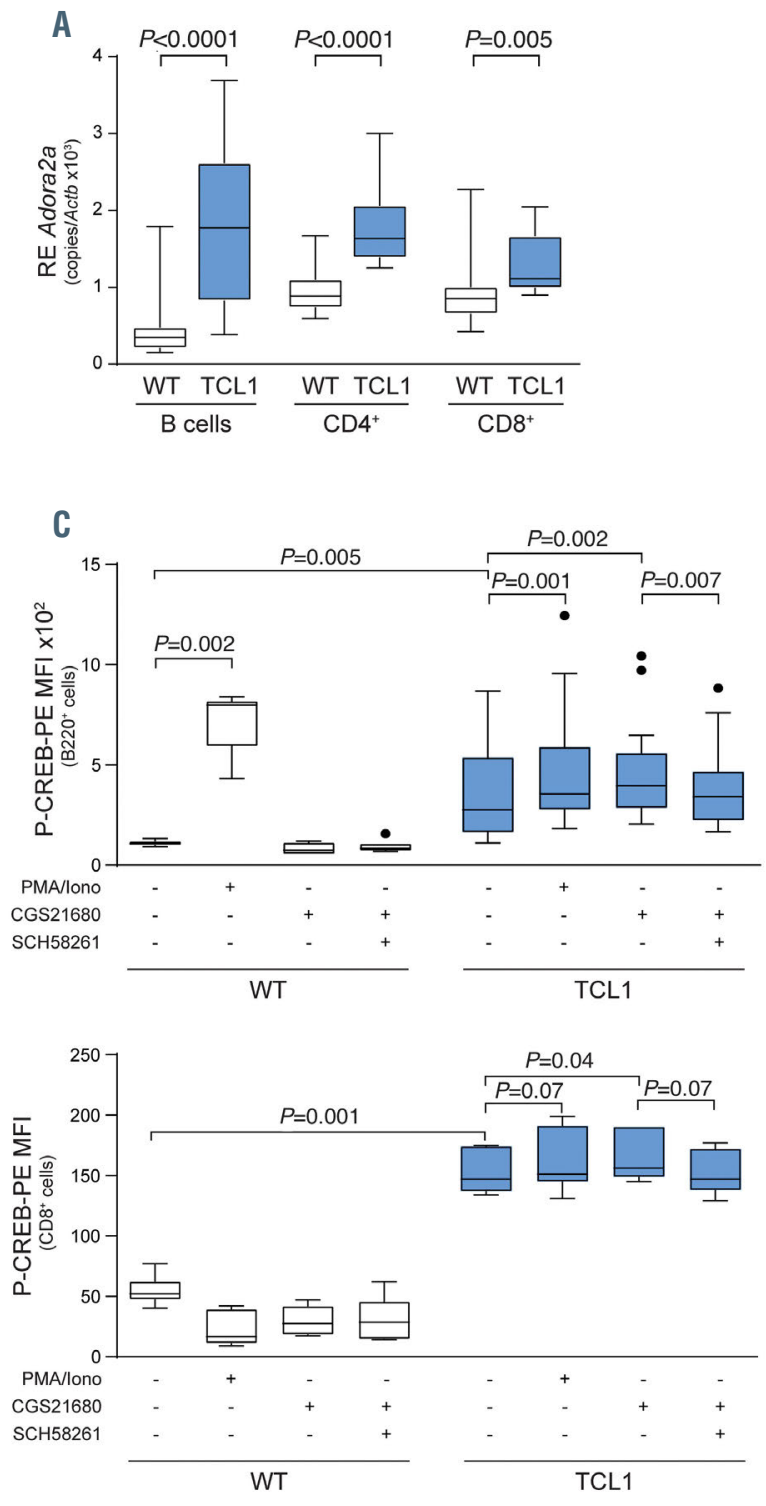


Figure 4. Upregulation of adenosine-sensing ability with leukemia progression. (A) Quantitative real-time polymerase chain reaction analysis of Adora2a expression in $B$ cells and in $\mathrm{CD}^{+}$or $\mathrm{CD}^{+}$T lymphocytes of TCL1 mice $(n=20)$ compared to the expression in wild-type (WT) animals ( $\left.n=20\right)$. Statistics were calculated with the Mann-Whitney test for unpaired data. RE: relative expression. (B) Confocal microscopy analysis of A2A upregulation in spleen sections of TCL1 mice at different stages of leukemia engraftment compared to WT mice. Magnification 63X. (C) Phosflow analysis of CREB phosphorylation in B-lymphocytes (top) or CD8 ${ }^{+}$cells (bottom) upon treatment with A2A agonist CGS21680 or A2A antagonist SCH58261. Data were compared between terminally ill TCL1 mice ( $\mathrm{n}=13$ ) and WT C57BL/6 mice $(n=6)$. Statistics were calculated using the Mann-Whitney test for unpaired data, when comparing TCL1 and WT mice, and the Wilcoxon test for paired data, when analyzing, within the same experimental group, the effect of stimulation/inhibition of A2A compared to the untreated condition. MFI: mean fluorescent intensity. (D) Enzyme-linked immunosorbent assay to evaluate protein kinase A (PKA) activity in B-lymphocytes from TCL1 mice compared to WT mice upon treatment with A2A agonist CGS21680 or A2A antagonist SCH58261. The results presented are from five independent experiments. Statistics were calculated using the Mann-Whitney test for unpaired data, when comparing TCL1 and WT mice, and the Wilcoxon test for paired data, when analyzing, within the same experimental group, the effect of stimulation/inhibition of A2A compared to the untreated condition. 
A

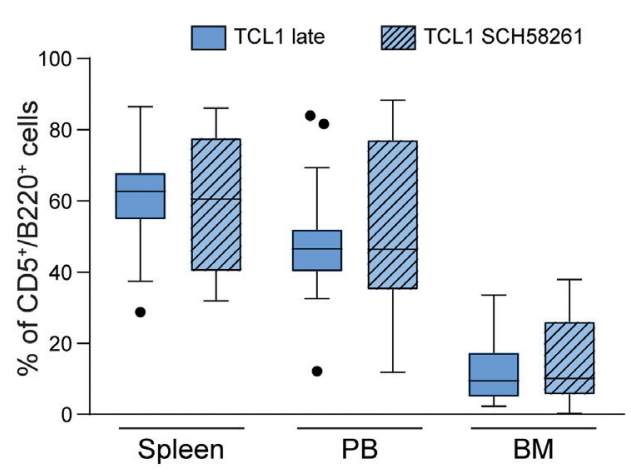

C

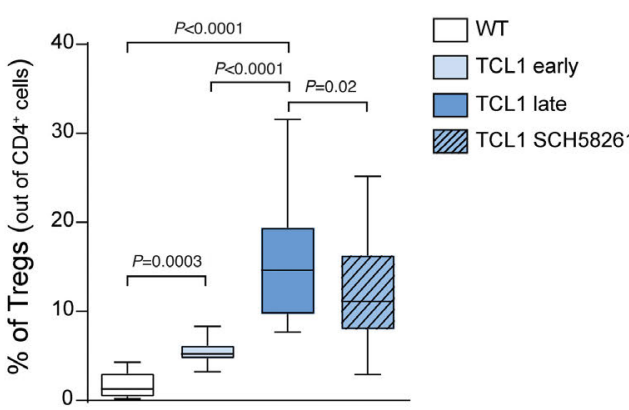

B

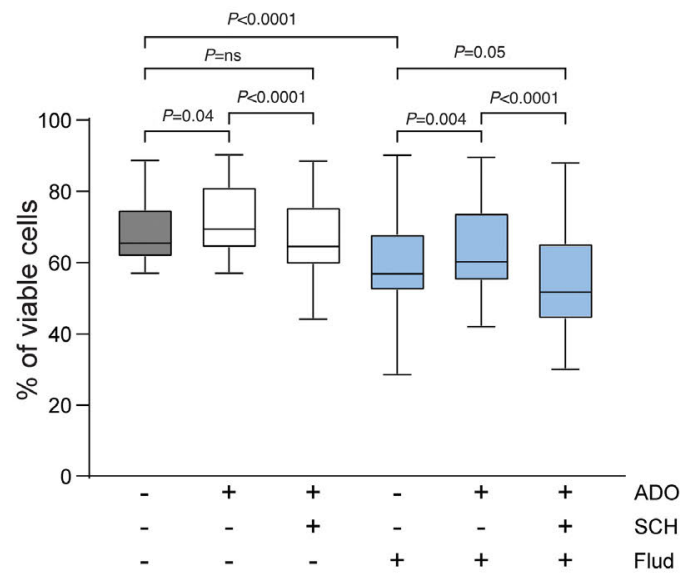

D
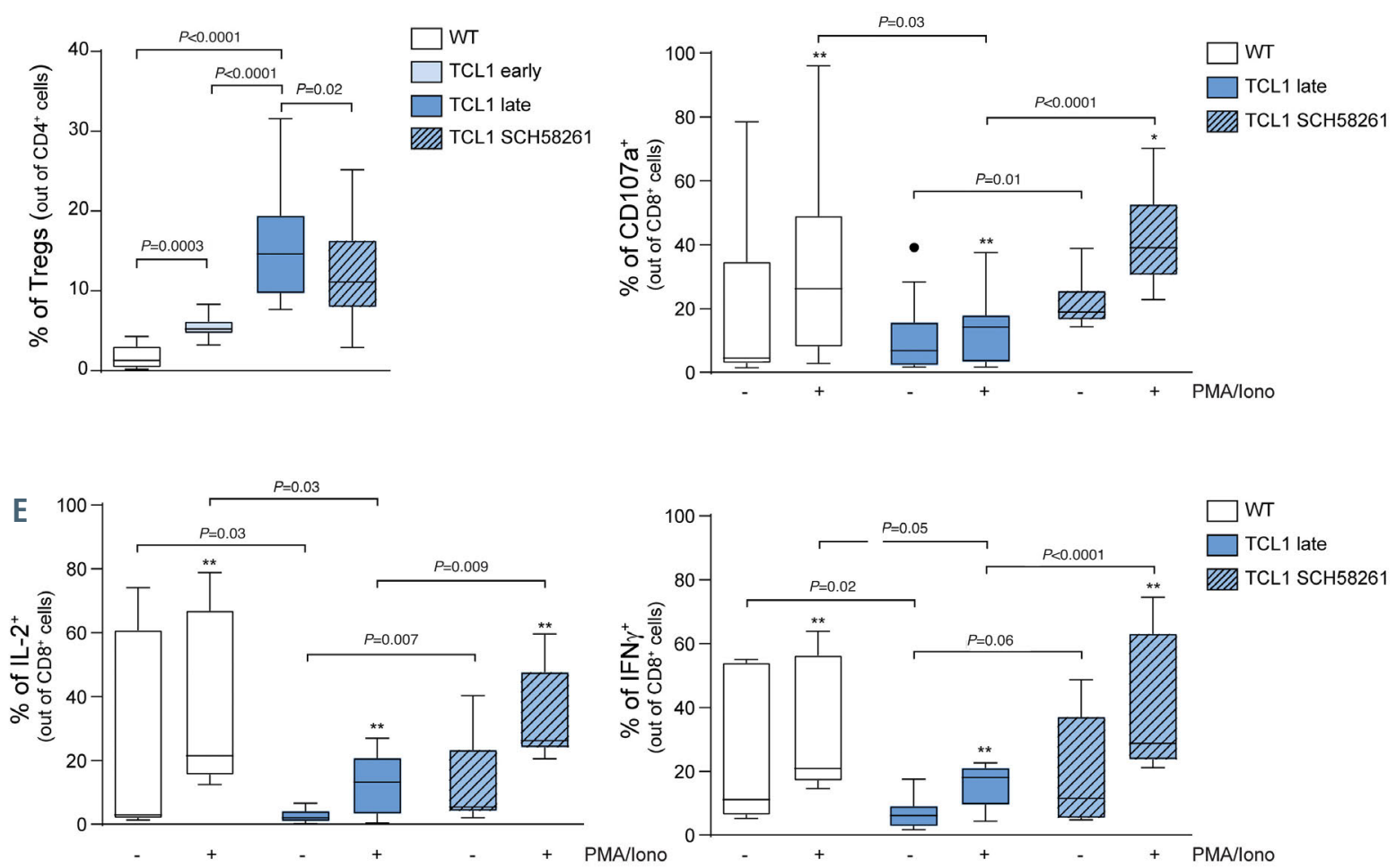

F

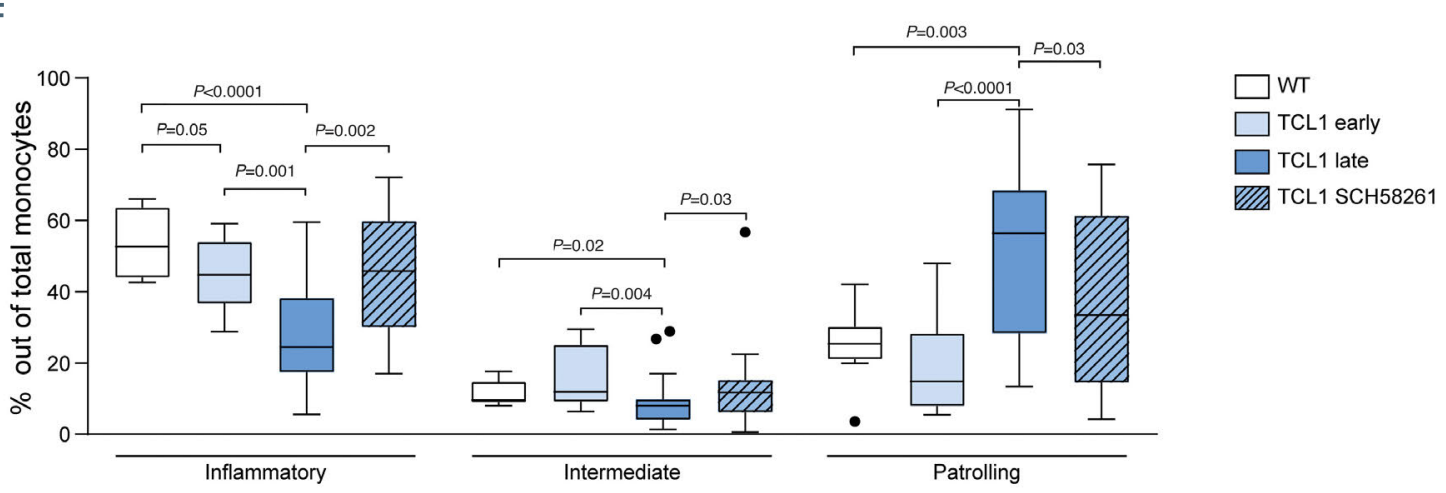

Figure 5. Legend on following page. 
Figure 5. Inhibition of A2A signaling in vivo partially restores immune competence. (A) Flow cytometry analysis of leukemic cells (defined as the CD5 $/$ B22 $^{+}$cell population) in the spleen, peripheral blood (PB) and bone marrow (BM) of TCL1 mice treated or not with SCH58261 ( $\mathrm{n}=20$ mice for each group). (B) In vitro effects of SCH58261 (SCH) on leukemic cell viability. Cells were cultured either alone or in the presence of $10 \mu \mathrm{M}$ fludarabine (Flud) for $48 \mathrm{~h}$. Where indicated, cells were supplied with $50 \mu \mathrm{M}$ adenosine (ADO) every $12 \mathrm{~h}$ or treated with $10 \mu \mathrm{M}$ SCH58261, starting from the beginning of the experiment and without replenishing it. ADO confers a survival advantage to TCL1 leukemic cells and protects them from spontaneous and fludarabine-induced apoptosis. SCH58261 efficiently reverts these effects without resulting per se in major apoptosis of leukemic cells, although a slight increase of fludarabine-induced apoptosis was observed in the presence of A2A inhibitor. Results are from 17 independent experiments and statistics were calculated with the Wilcoxon test for paired data. (C) Percentage of regulatory T cells (Treg) out of the total CD4 ${ }^{+}$cell population progressively increases in TCL1 mice, analyzed at different time-points after injection of leukemic cells, compared to WT not injected mice. In vivo treatment with SCH58261 significantly prevents Treg differentiation. Analyses were performed on 20 TCL1 mice for each condition (different time-points and SCH58261 treatment) compared to seven WT not injected mice. Statistics were calculated using the Mann-Whitney test for unpaired data. (D) Percentage of $C D 107 a^{+}$cells out of $\mathrm{CD}^{+} \mathrm{T}$ lymphocytes in terminally ill vehicle-treated TCL1 mice $(\mathrm{n}=12)$ compared to SCH58261-treated mice $(\mathrm{n}=8)$ or to WT C57BL/6 $(n=12)$. Statistics were calculated using the Mann-Whitney test for unpaired data, when comparing TCL1 and WT mice, and the Wilcoxon test for paired data, when analyzing, within the same experimental group, the effect of stimulation/inhibition of A2A compared to the untreated condition. (E) Percentage of interleukin (IL)- $2^{+}$(left panel) and interferon (IFN)- $\gamma^{+}$(right panel) cells out of CD8 ${ }^{+} \mathrm{T}$ lymphocytes in terminally ill vehicle-treated TCL1 mice ( $\left.\mathrm{n}=12\right)$ compared to SCH58261 treated mice $(n=8)$ or to WT C57BL/6 $(n=12)$. Statistics were calculated using the Mann-Whitney test for unpaired data, when comparing TCL1 and WT mice, and the Wilcoxon test for paired data, when analyzing, within the same experimental group, the effect of stimulation/inhibition of A2A compared to the untreated condition. (F) Characterization of monocyte subpopulations in TCL1 mice, analyzed at different time-points after injection of leukemic cells, compared to WT not injected mice, show a dramatic increase of the patrolling (M2-like) subset at the expense of the inflammatory (M1-like) one. In vivo treatment with SCH58261 significantly rescues skewing of monocytes, restoring the inflammatory subset. Monocytes were defined as the Lin-CD $11 \mathrm{~b}^{+} \mathrm{F} 4 / 80^{+}$cell fraction and subpopulations were identified on the basis of LY6C and CD43 expression (inflammatory: $\mathrm{LY}^{2} \mathrm{C}^{+} \mathrm{CD} 43^{-}$; intermediate: $\mathrm{LY} 6 \mathrm{C}^{+} \mathrm{CD} 43^{+}$; patrolling: $\mathrm{LY}^{-} \mathrm{C}^{-} \mathrm{CD} 43^{+}$). Analyses were performed on 20 TCL1 mice for each condition (different time-points and SCH58261 treatment) compared to seven WT not injected mice. Statistics were calculated using the MannWhitney test for unpaired data.

response element binding protein (CREB). ${ }^{17,18}$ For this reason, we set-up a phosflow assay to rapidly assess CREB phosphorylation status upon exposure of different cell subsets to A2A agonists. The results indicated that leukemic cells, as well as CD4 and CD8 subsets, showed constitutively higher levels of CREB phosphorylation, likely indicating an activated status of the pathway. Addition of CGS, a specific A2A agonist, further enhanced CREB phosphorylation, suggesting that in this context inflammatory responses are blunted. As a control, cells were pre-treated with the A2A antagonist SCH58261 before exposure to the agonist, completely inhibiting CREB phosphorylation (Figure 4C, Online Supplementary Figure S2C and D). These data were confirmed on leukemic cells by demonstrating PKA activation, which was higher in leukemic B cells than in normal B cells, further enhanced by the agonist and blocked by the antagonist (Figure 4D).

\section{In vivo targeting of $\mathrm{A} 2 \mathrm{~A}$ restores immune competence}

Data so far indicate that progression of leukemia is accompanied by increased hypoxia, which leads to the modulation of the adenosinergic axis, with production of adenosine and activation of its signaling pathway. The hypothesis is that this may lead to progressive adaptation of the immune system to these conditions, with inhibition of potentially tumor-specific T cells. If this is true, inhibition of A2A signaling should prove beneficial to immune cells, reconstituting their functional properties.

In order to address this issue, we treated leukemic-bearing mice with the commercially available SCH58261 A2A antagonist, which was shown to be well tolerated in animals. The A2A antagonist was administered every other day for 2 weeks, starting from 4 days after injection of the leukemic cells. Sixteen hours after the last administration, animals were euthanized and leukemia dissemination as well as immune cell populations were analyzed.

In line with our previous data showing that extracellular adenosine exerts mild protection from drug-induced apoptosis on leukemic cells, ${ }^{19}$ SCH58261 treatment in monotherapy in vivo had minimal effects on tumor burden, with no significant differences highlighted between vehicle- or SCH58261-treated mice when looking at the percentage of leukemic cells colonizing the spleen, peripheral blood or bone marrow (Figure 6B). Similar to what was observed in primary human CLL cells, in vitro expo- sure of TCL1 leukemic cells to adenosine significantly improved cell viability in the presence of standard chemotherapeutic agents (e.g., fludarabine). Blocking A2A signaling with SCH58261 reverted the cytoprotective effect of adenosine but did not result in a major apoptotic response of TCL1 leukemic cells when used as a single agent. In contrast, it slightly but significantly potentiated the antileukemic effect of fludarabine, opening the way to combination studies (Figure 6A).

The administration of SCH58261 as monotherapy was however very useful for identifying the major contribution of A2A signaling in leukemia progression in vivo. In fact, as expected, leukemia progression was accompanied by marked immune adaptation, as documented by the significant increase in regulatory $\mathrm{T}$ cells (Treg), a finding also in line with what was observed in patients. ${ }^{20}$ Treatment with SCH58261 prevented accumulation of Treg; the percentage of Treg out of total $\mathrm{CD}^{+}$cells was $15.6 \%$ in terminally ill vehicle-treated mice versus $11.8 \%$ in SCH58261-treated mice (Figure 5A, Online Supplementary Figure S3A). In addition, T cells from leukemic mice were dysfunctional, showing reduced expression of the degranulation marker CD107a (Figure 5B, Online Supplementary Figure $S 3 B$ ), an effect again reverted upon A2A blockade. $T$ cells from terminally ill mice also secreted significantly less interleukin-2 and interferon- $\gamma$ compared to cells obtained from WT animals, both at baseline and after short-term incubation with phorbol 12-myristate 13acetate (PMA) and ionomycin (Figure 5C, Online Supplementary Figure $S 3 B$ ), again in line with data from patients. ${ }^{21}$ Compared to the $\mathrm{T}$ cells from vehicle-treated TCL1 mice, those from mice given SCH58261 showed restored ability to secrete interleukin- 2 and interferon- $\gamma$, both at baseline and following maximal cellular activation with PMA and ionomycin (Figure 5C, Online Supplementary Figure S3B). Lastly, treatment with SCH58261 had a notable impact on the myeloid subset of cells. Indeed, leukemia progression was marked by an increase in patrolling monocytes at the expense of inflammatory ones, suggesting an imbalance similar to that observed in patients. ${ }^{22}$ Importantly, inhibition of A2A signaling reverted this adaptation, restoring the inflammatory component and limiting the patrolling one (Figure $5 \mathrm{D}$, Online Supplementary Figure S3C).

The results suggest that targeting adenosine signaling 



Figure 6. Effects of adenosine signaling and A2A inhibition. Hypoxia upregulates CD39 and CD73 leading to increased adenosine generation. Adenosine in the extracellular space signals mainly through the A2A receptor, expressed on leukemic cells as well as immune cells in the microenvironment, and exerts autocrine and paracrine effects. Signaling via A2A activates protein kinase A (PKA) and CREB phosphorylation, which in turn inhibits nuclear factor (NF)- $\mathrm{B} B$-driven inflammation. Depending on the cell population, A2A signaling may favor different outcomes each contributing to an immune-tolerant environment, such as interleukin (IL)-10 secretion by chronic lymphocytic leukemia (CLL) cells, regulatory T cell (Treg) differentiation and expansion, exhaustion of CD8 ${ }^{+} \mathrm{T}$ cells, and monocytes switching toward an M2 (patrolling) phenotype. Inhibition of A2A signaling with SCH58261 rescues CD8 ${ }^{+}$cytotoxicity and ability to secrete cytokines, such as IL-2 and interferon (IFN)- $\gamma$, while limiting Treg expansion and favoring monocyte differentiation towards a M1 (inflammatory) phenotype.

might be particularly relevant when interfering with paracrine circuits affecting immune cell functions, rather than having an impact on CLL cytoprotection. In addition, the observation that in SCH58261-treated mice no correlation was found between the variation of the tumor loads and the size of the effect of immune system repolarization (Online Supplementary Figure S4A-D) suggests that blocking A2A signaling might be sufficient to rescue immune cell dysfunctions, even without evidence of tumor regression. These findings prompt the way to future drug combination studies aimed at dually targeting central pathways for the malignant behavior of leukemic cells while restoring immune competence, in order to achieve the best longterm responses.

\section{Discussion}

Dysfunction of the immune system is a major clinical issue in CLL since the early stages of the disease predispose patients to recurrent infections, which represent a major cause of morbidity and mortality. ${ }^{23}$ Qualitative and quantitative defects in the innate and adaptive immune responses are frequently found in CLL patients and include T-cell exhaustion, expansion of Treg and skewing of macrophages towards a tumor-supportive M2 phenotype..$^{21,22,24,25}$ Growing evidence indicates that leukemic cells primarily drive immune cell dysregulation in an attempt to evade immune control. For example, CLL cells show features of regulatory $\mathrm{B}$ cells and they might exert a direct immunosuppressive effect by secreting interleukin-10. ${ }^{26}$ Regulatory activity is further enhanced within the lymphoid niche, likely as a consequence of local B-cell activation, highlighting the importance of microenvironmental conditions in bidirectional tumor-host remodeling. ${ }^{27}$

The TCL1 adoptive transfer model of CLL is based on injection of leukemic cells obtained from C57BL/6 animals that are transgenic for TCL1 into syngeneic hosts. ${ }^{28}$ Leukemic cells injected into the peritoneum engraft and leukemia develops over a period of a few weeks, during which it is possible to observe progressive adaptation of the immune system. Specifically, naive $\mathrm{CD}^{+}{ }^{+}$and $\mathrm{CD} 8^{+} \mathrm{T}$ lymphocytes are progressively substituted by memory cells that bear exhaustion markers and are poorly responsive to activation. In addition, the regulatory T-cell pool expands, while in the myeloid compartment the inflammatory subset is substituted by patrolling cells. ${ }^{29.32}$ As these 
events are time-dependent and highly reproducible, they are sensitive tools for studying immune adaptation.

This work shows that leukemia progression is accompanied by progressive tissue hypoxia, which in turn powerfully modulates the adenosinergic system. Modulation of the HIF-1 $\alpha$ pathway was documented both by increased expression of the carbonic anhydrase IX protein, which is under its direct transcriptional control, and by increased staining with PIM, which binds macromolecules specifically under low oxygen tension. We previously demonstrated that, under hypoxia, CLL cells undergo HIF-1 $\alpha$-driven reprogramming, which favors metabolic adaptation and blunting of the immune response, partly through the upregulation of the adenosinergic axis. ${ }^{14}$

Leukemia progression is also accompanied by increased expression of the enzymes that are responsible for adenosine conversion from ATP or ADP in the extracellular space. CD39, which is also considered a marker of memory $B$ cells, becomes expressed by the great majority of leukemic cells. The same trend is observed for CD73, which is the rate-limiting enzyme in the pathway. Immunohistochemical analysis also confirmed a general upregulation of CD73 expression with the progression of leukemia.

Extracellular adenosine can, therefore, exert both autocrine and paracrine effects, similarly to what was described in human CLL. ${ }^{19}$ On the one hand, the observation that murine leukemic cells also strongly upregulate adenosine deaminase suggests that adenosine is in part recycled via conversion to inosine. On the other hand, more importantly, we found an overall increase of adenosine receptor expression and signaling in the spleens of terminally ill mice, with A2A being the most expressed receptor. This was documented at the mRNA level both on leukemic $\mathrm{B}$ cells and on $\mathrm{CD}^{+}$and $\mathrm{CD} 8^{+} \mathrm{T}$ lymphocytes purified from TCL1 mice and compared to those from WT C57BL/6 mice, in line with the view that the adenosinergic axis is part of a hypoxia-driven transcriptional reprogramming. Consistently, spleen sections of leukemic mice stained much more strongly positive for A2A compared to WT spleen. The finding that A2A signaling, via PKA activation and CREB phosphorylation, was strongly increased in $B$ and $T$ cells from terminally ill mice, compared to control animals, argues in favor of an adenosine-mediated circuit, activated by $\mathrm{HIF}-1 \alpha$, responsible, at least in part, for immunosuppression. In fact, in human B cells, PKA-mediated CREB activation induces interleukin-10 production and secretion, contributing to an anti-inflammatory immunetolerant environment and directly linking A2A signaling to immunomodulation. ${ }^{14,18}$ Consistent with this view, and with previous literature, we observed a progressive dysregulation of the immune system in leukemic mice, affecting Tcell functions and monocyte polarization. Restoring immune competence represents a major achievement in CLL management and here we explored the effects of targeting the adenosinergic axis and its potential application in a translational perspective. Importantly, we show that interfering with adenosine signaling, using a specific A2A inhibitor, significantly rescues $\mathrm{CD}^{+} \mathrm{T}$-cell responses and functions while preventing the expansion of immunomodulatory subsets such as Treg and patrolling monocytes.
The establishment of a progressively hypoxic environment promotes metabolic adaptation and reprogramming in CLL, contributing also to enhance leukemic cell aggressiveness and to inducing drug resistance. ${ }^{33}$ Adenosine signaling mediates part of these effects, favoring metabolic switching of CLL cells and conferring them cytoprotection and immune regulatory functions. ${ }^{14}$ Nevertheless, our results also indicated that SCH58261 treatment in vivo had little or no effect on leukemic cells, suggesting that paracrine, rather than autocrine, effects of extracellular adenosine play an important role in disease progression, at least in this experimental setting (Figure 7), arguing in favor of using A2A inhibitors as part of combination strategies. Novel, more specific and efficient A2A inhibitors are currently under development, and future studies are coming to validate their effects both as single agents and in combination.

The lesson from data on solid tumors, ${ }^{34}$ and further substantiated by our results, is that targeting the adenosinergic axis could be a promising strategy to re-awake the immune system and restore immune-cell functions. Applied to the field of CLL, these data might be a starting point to design and develop more effective therapies. Different possible scenarios can be envisaged: one option could be to combine inhibitors of the adenosinergic cascade together with drugs directly targeting central pathways of the CLL cell. Alternatively, targeting adenosine signaling may represent an adjunctive tool to potentiate chimeric antigen receptor T-cell activity against CLL cells, also considering the promising results obtained with this novel therapeutic approach. ${ }^{35}$ Further studies will tell which of these avenues will be more promising.

\section{Disclosures}

No conflicts of interest to disclose.

\section{Contributions}

FA designed the study, performed experiments, analyzed and interpreted data and together with $T V$ and $S D$ wrote the paper; $S S$ and $A P$ designed the study and performed experiments; NV, GG, BBG and FT performed experiments; DE provided vital reagents; TV and SD designed the study, analyzed and interpreted data and wrote the paper.

\section{Acknowledgments}

We thank Dr. Denis Baev for excellent support with the design of the flow cytometry panel and with the data analysis.

\section{Funding}

This work was supported by Italian Institute for Genomic Medicine institutional funds, by the Associazione Italiana per la Ricerca sul Cancro AIRC (IG17314 to SD), by the Gilead Fellowship Program 2018 (by the ITN INTEGRATA program, grant agreement 813284 to SD), by the Italian Ministry of Health (GR-2016-02364298 to TV), by the Ministry of Education, University and Research-MIUR "Progetto Strategico di Eccellenza Dipartimentale" (\#D15D18000410001 to SD in part through funds to the Department of Medical Sciences, University of Turin); and by the Ministry of Education, University and Research-MIUR PRIN project (2017CBNCYT to $S D)$. 


\section{References}

1. Zhang S, Kipps TJ. The pathogenesis of chronic lymphocytic leukemia. Annu Rev Pathol. 2014;9:103-118.

2. Burger JA, Gribben JG. The microenvironment in chronic lymphocytic leukemia (CLL) and other B cell malignancies: insight into disease biology and new targeted therapies. Semin Cancer Biol. 2014;24:71-81.

3. Sutton LA, Rosenquist R. The complex interplay between cell-intrinsic and cellextrinsic factors driving the evolution of chronic lymphocytic leukemia. Semin Cancer Biol. 2015;34:22-35.

4. ten Hacken E, Burger JA.Microenvironment dependency in chronic lymphocytic leukemia: the basis for new targeted therapies. Pharmacol Ther. 2014; 144(3):338-348.

5.ten Hacken E, Burger JA. Microenvironment interactions and B-cell receptor signaling in chronic lymphocytic leukemia: implications for disease pathogenesis and treatment. Biochim Biophys Acta. 2016;1863(3):401-413.

6. Barrientos JC. Sequencing of chronic lymphocytic leukemia therapies. Hematology Am Soc Hematol Educ Program. 2016; 2016(1):128-136

7. Arruga F, Deaglio S. Mechanisms of resistance to targeted therapies in chronic lymphocytic leukemia. Handb Exp Pharmacol. 2018;249:203-229.

8. van Attekum MH, Eldering E, Kater AP. Chronic lymphocytic leukemia cells are active participants in microenvironmental cross-talk. Haematologica. 2017; 102(9): 1469-1476.

9. Tsukada N, Burger JA, Zvaifler NJ, Kipps TJ. Distinctive features of "nurselike" cells that differentiate in the context of chronic lymphocytic leukemia. Blood. 2002; 99(3):1030-1037

10. Wilson WR, Hay MP. Targeting hypoxia in cancer therapy. Nat Rev Cancer. 2011;11(6):393-410.

11. Valsecchi R, Coltella N, Belloni D, et al. HIF-1 $\alpha$ regulates the interaction of chronic lymphocytic leukemia cells with the tumor microenvironment. Blood. 2016; 127(16): 1987-1997

12. Bhandari V, Hoey C, Liu LY, et al. Molecular landmarks of tumor hypoxia across cancer types. Nat Genet. 2019;51(2):308-318.

13. Koczula KM, Ludwig C, Hayden R, et al. Metabolic plasticity in CLL: adaptation to the hypoxic niche. Leukemia. 2016; 30(1):65-73.

14. Serra S, Vaisitti T, Audrito V, et al. Adenosine signaling mediates hypoxic responses in the chronic lymphocytic leukemia microenvironment. Blood Adv. 2016;1(1):47-61.

15. Beavis PA, Divisekera U, Paget C, et al. Blockade of A2A receptors potently suppresses the metastasis of CD73+ tumors. Proc Natl Acad Sci U S A. 2013;110(36): 14711-14716

16. Serra S, Deaglio S. HPLC-based assay to monitor extracellular nucleotide/nucleoside metabolism in human chronic lymphocytic leukemia cells. J Vis Exp. 2016;(113). doi: $10.3791 / 54124$

17. Klinger $M$, Freissmuth $M$, Nanoff C. Adenosine receptors: $G$ protein-mediated signalling and the role of accessory proteins. Cell Signal. 2002;14(2):99-108.

18. Wen AY, Sakamoto KM, Miller LS. The role of the transcription factor CREB in immune function. J Immunol. 2010;185(11):64136419.

19. Serra S, Horenstein AL, Vaisitti T, et al. CD73-generated extracellular adenosine in chronic lymphocytic leukemia creates local conditions counteracting drug-induced cell death. Blood. 2011;118(23):6141-6152.

20. Jak M, Mous R, Remmerswaal EB, et al. Enhanced formation and survival of CD4+ CD25hi Foxp3+ T-cells in chronic lymphocytic leukemia. Leuk Lymphoma. 2009; 50(5):788-801.

21. Riches JC, Davies JK, McClanahan F, et al. $\mathrm{T}$ cells from CLL patients exhibit features of T-cell exhaustion but retain capacity for cytokine production. Blood. 2013; 121(9):1612-1621.

22. Jitschin $R$, Braun $M$, Buttner $M$, et al. CLLcells induce IDOhi CD14+HLA-DRlo myeloid-derived suppressor cells that inhibit T-cell responses and promote TRegs. Blood. 2014;124(5):750-760.

23. Teh BW, Tam CS, Handunnetti S, Worth LJ, Slavin MA. Infections in patients with chronic lymphocytic leukaemia: mitigating risk in the era of targeted therapies. Blood Rev. 2018;32(6):499-507.

24. Audrito V, Serra S, Brusa D, et al. Extracellular nicotinamide phosphoribosyltransferase (NAMPT) promotes M2 macrophage polarization in chronic lymphocytic leukemia. Blood. 2015;125(1):111-123.

25. Forconi F, Moss P. Perturbation of the nor- mal immune system in patients with CLL. Blood. 2015;126(5):573-581.

26. DiLillo DJ, Weinberg JB, Yoshizaki A, et al. Chronic lymphocytic leukemia and regulatory B cells share IL-10 competence and immunosuppressive function. Leukemia. 2013;27(1):170-182.

27. Ringelstein-Harlev S, Avivi I, Fanadka M, Horowitz NA, Katz T. Chronic lymphocytic leukemia cells acquire regulatory B-cel properties in response to TLR9 and CD40 activation. Cancer Immunol Immunother. 2018;67(5):739-748.

28. Zanesi N, Aqeilan R, Drusco A, et al. Effect of rapamycin on mouse chronic lymphocytic leukemia and the development of nonhematopoietic malignancies in EmuTCL1 transgenic mice. Cancer Res. 2006; 66(2):915-920.

29. Hofbauer JP, Heyder C, Denk U, et al Development of CLL in the TCL1 transgenic mouse model is associated with severe skewing of the T-cell compartment homologous to human CLL. Leukemia. 2011;25(9):1452-1458.

30. McClanahan F, Hanna B, Miller S, et al. PDL1 checkpoint blockade prevents immune dysfunction and leukemia development in a mouse model of chronic lymphocytic leukemia. Blood. 2015;126(2):203-211.

31. Hanna BS, McClanahan F, Yazdanparast H, et al. Depletion of CLL-associated patrolling monocytes and macrophages controls disease development and repairs immune dysfunction in vivo. Leukemia. 2016;30(3):570-579

32. Hanna BS, Roessner PM, Yazdanparast H, et al. Control of chronic lymphocytic leukemia development by clonally-expanded CD8(+) T-cells that undergo functional exhaustion in secondary lymphoid tissues. Leukemia. 2019;33(3):625-637.

33. Guieze R, Liu VM, Rosebrock D, et al. Mitochondrial reprogramming underlies resistance to BCL-2 inhibition in lymphoid malignancies. Cancer Cell. 2019;36(4):369384 e313.

34. Young A, Ngiow SF, Barkauskas DS, et al Co-inhibition of CD73 and A2AR adenosine signaling improves anti-tumor immune responses. Cancer Cell. 2016; 30(3):391-403.

35. Lemal R, Tournilhac O. State-of-the-art for CAR T-cell therapy for chronic lymphocytic leukemia in 2019. J Immunother Cancer 2019;7(1):202. 